\title{
p300 is upregulated by docetaxel and is a target in chemoresistant prostate cancer
}

\author{
Martina Gruber1, Lavinia Ferrone1,2, Martin Puhr1, Frédéric R Santer', Tobias Furlan1, Iris E Eder', Natalie Sampson'1, \\ Georg Schäfer ${ }^{3}$, Florian Handle ${ }^{1,4}$ and Zoran Culig1
}

1Division of Experimental Urology, Department of Urology, Medical University of Innsbruck, Innsbruck, Austria 2Department of Biomedical, Experimental and Clinical Sciences, University of Florence, Florence, Italy

3Department of Pathology, Neuropathology, and Molecular Pathology, Medical University of Innsbruck, Innsbruck, Austria

${ }^{4}$ Molecular Endocrinology Laboratory, Department of Cellular and Molecular Medicine, KU Leuven, Leuven, Belgium

Correspondence should be addressed to Z Culig: zoran.culig@i-med.ac.at

\begin{abstract}
Administration of the microtubule inhibitor docetaxel is a common treatment for metastatic castration-resistant prostate cancer (mCRPC) and results in prolonged patient overall survival. Usually, after a short period of time chemotherapy resistance emerges and there is urgent need to find new therapeutic targets to overcome therapy resistance. The lysine-acetyltransferase p300 has been correlated to prostate cancer (PCa) progression. Here, we aimed to clarify a possible function of p300 in chemotherapy resistance and verify p300 as a target in chemoresistant PCa. Immunohistochemistry staining of tissue samples revealed significantly higher p300 protein expression in patients who received docetaxel as a neoadjuvant therapy compared to control patients. Elevated p300 expression was confirmed by analysis of publicly available patient data, where significantly higher p300 mRNA expression was found in tissue of mCRPC tumors of docetaxel-treated patients. Consistently, docetaxel-resistant PCa cells showed increased p300 protein expression compared to docetaxel-sensitive counterparts. Docetaxel treatment of PCa cells for $72 \mathrm{~h}$ resulted in elevated $\mathrm{p} 300$ expression. shRNAmediated p300 knockdown did not alter colony formation efficiency in docetaxelsensitive cells, but significantly reduced clonogenic potential of docetaxel-resistant cells. Downregulation of p300 in docetaxel-resistant cells also impaired cell migration and invasion. Taken together, we showed that p300 is upregulated by docetaxel, and our findings suggest that p300 is a possible co-target in treatment of chemoresistant PCa.
\end{abstract}

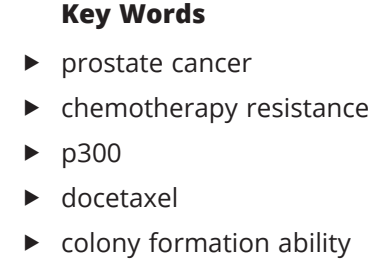

Key Words

- prostate cancer

- chemotherapy resistance

- p300

- colony formation ability

\section{Introduction}

Although therapy of organ-confined prostate cancer (PCa) by radical prostatectomy or radiotherapy is curative, treatment of advanced PCa is considered merely palliative. Androgen deprivation therapy (ADT) remains the gold standard for patients with prostate-specific antigen (PSA) progression. However, tumor progression is inevitable and leads to the development of castration-resistant prostate cancer (CRPC). Treatment options for non-metastatic and metastatic CRPC (mCRPC) include inhibitors of androgen synthesis and antiandrogens such as enzalutamide and apalutamide as well as the chemotherapeutic compound docetaxel (Taxotere $\left.{ }^{\circledR}\right)$.

In 2004, the landmark study TAX327 showed a significant survival benefit of 2.4 months for docetaxel
(C) 2020 The authors Published by Bioscientifica Ltd. Printed in Great Britain
Endocrine-Related Cance (2020) 27, 187-198 
treatment compared to mitoxantrone, which was the first study to demonstrate a survival benefit for chemotherapy in CRPC patients (Tannock et al. 2004). Docetaxel treatment resulted in PSA decline, prolonged overall survival (OS), and improved quality of life. Furthermore, the STAMPEDE and CHAARTED trials have utilized docetaxel together with ADT into first-line treatment for hormone sensitive PCa (HSPC) with a survival benefit of 13.4 months compared to ADT alone (James et al. 2016, Kyriakopoulos et al. 2018). Additionally, docetaxel treatment has no negative consequences for subsequent endocrine therapies. Both abiraterone acetate and enzalutamide are used as effective second-line therapies after resistance to docetaxel has evolved (Lavaud et al. 2018). Despite development of novel therapies, treatment options for mCRPC patients are still limited, and there is an urgent need to find new therapeutic targets to overcome chemotherapy resistance.

Transcriptional co-regulators of the AR are involved in therapy resistance with several of them increasingly expressed during ADT (Comuzzi et al. 2004, Heemers et al. 2007, Qin et al. 2014). Two of these well-known coactivators are the histone acetyltransferases p300 and CBP (CREB binding protein) that show elevated expression in advanced PCa and have oncogenic potential (Debes et al. 2003, Comuzzi et al. 2004). While these coactivators show high levels of homology, they play distinctive roles in PCa and other diseases (Kalkhoven 2004, Ramos et al. 2010). Common features of p300 and CBP include regulation of transcription via remodeling chromatin structure by acetylating conserved lysine amino acids of histone proteins. They are also capable of recruiting the basal transcription machinery to gene promoters and acting as adaptor molecules (Chan \& La Thangue 2001). In a previous study, it was demonstrated that p300 might be a valid target in PCa cells, as downregulation of p300 induced apoptosis and decreased cell migration in androgen-dependent and CRPC cells (Santer et al. 2011). Based on those previous results, this study aimed to investigate whether p300 is a possible new target in the context of chemotherapy resistance. Therefore, we analyzed (1) p300 expression in patients that received docetaxel, (2) p300 expression in docetaxelresistant (DR) cells compared to docetaxel-sensitive counterparts, and (3) the effects of short-term docetaxel treatment on p300 expression. To study the functional role of p300, an RNA-interference (RNAi) approach was used and doxycycline-inducible p300 knockdown cell lines were generated. In addition, effects of p300 downregulation on colony formation efficiency, cell migration, and invasion in docetaxel-resistant cells were determined.

\section{Materials and methods}

\section{Cell culture and chemicals}

Human PCa cell lines PC3 and DU145 were purchased from the American Type Culture Collection (ATCC, LGC Standards, Wesel, Germany). Docetaxel-resistant PC3-DR and DU145-DR were previously established by Puhr et al. (2012). CWR22RV1 and CWR22RV1-DR cells were a kind gift of Prof Dr William Watson (University College Dublin). All cell lines were cultured in RPMI 1640 (PAN Biotech, Aidenbach, Germany) supplemented with $10 \%$ (v/v) fetal bovine serum (PAN Biotech, Aidenbach, Germany), $1 \%(\mathrm{v} / \mathrm{v})$ penicillin/streptomycin, and 1\% (v/v) GlutaMAX (both from Lonza, Vienna, Austria). Docetaxel-resistant cell lines were cultured in the presence of $12.5 \mathrm{nmol} / \mathrm{L}$ docetaxel (Sigma Aldrich). HEK293FT cells were obtained from Life Technologies and grown according to the manufacturer's instructions. The authenticity of all cell lines was validated via short tandem repeat (STR) profiling.

\section{Immunohistochemistry (IHC)}

For IHC staining, a tissue microarray (TMA) of 14 patients that received neoadjuvant docetaxel therapy before radical prostatectomy and 14 patients with no chemotherapy was used. The use of patient material was approved by the Ethics Committee of the Medical University of Innsbruck (study No AM 3174 including amendment 2). For detailed information about clinical data from patients, see publication of Puhr et al. (2012). IHC staining was performed on a Discovery-XT staining device (Ventana) and the following specific antibody was used: anti-p300 (1:100, D8Z4E, Cell Signaling Technology). Antibody specificity was verified by Western blot and IHC staining of PC3-DR cells with p300 downregulation. For IHC, cells were embedded by coagulation in plasma clots after harvesting, transferred into a biopsy histosette, fixed in formalin, and embedded in paraffin. Importantly, cross staining of CBP was excluded by Western blot analysis.

\section{Transcriptome analysis of patient data}

The publicly available transcriptome dataset GSE77930 (Kumar et al. 2016) was downloaded from the GEO database and analyzed with the Qlucore Omics Explorer v3.5. Gene set activity scores for the Hallmark 'Androgen response' and 'Myc targets' gene sets (Molecular Signatures Database, MSigDB) were calculated in $\mathrm{R}$ with the GSVA package (Hanzelmann et al. 2013).

This work is licensed under a Creative Commons Attribution 4.0 International License. ded from Bioscientifica.com at 04/26/2023 09:57:30AM 


\section{Western blot}

Cells were lysed in LDS sample buffer and $50 \mu \mathrm{g}$ total protein was separated either on 3-8\% Tris-Acetate gels (Thermo Fisher Scientific) for analysis of p300 and CBP expression or $4-12 \%$ Bis-Tris gels (Expedeon, San Diego, CA, USA) for all other proteins and transferred onto methanol-activated PVDF membranes or $0.2 \mu \mathrm{m}$ nitrocellulose membranes (GE Healthcare). Blocking of membranes and antibody incubation were performed in 5\% BSA in TBS-T. The following antibodies were used: anti-p300 (1:4000, ab10485, Abcam), anti-CBP (1:1000, Cell Signaling Technology), antic-Myc (1:1000, Cell Signaling Technology), anti-Histone H3 (1:1000, Cell Signaling Technology), anti-Acetyl-Histone H3 (Lys18, 1:1000, Cell Signaling Technology), anti-Vimentin (1:500, Santa Cruz Biotechnology), anti-Vinculin (1:500, Santa Cruz Biotechnology), anti-Lamin A (1:2000, Abcam), anti- $\alpha$-tubulin (1:500, Santa Cruz Biotechnology), and antiGAPDH (1:50000, Merck Millipore). House-keeping controls were selected in a cell line-specific manner on the basis of data showing no change in their expression in that cell line.

\section{RNA isolation and quantitative real-time PCR}

Total RNA was isolated using the EXTRACTME TOTAL RNA KIT (LabConsulting, Vienna, Austria) according to the manufacturer's manual. cDNA synthesis was performed with the iScript Select cDNA Synthesis Kit (Bio-Rad). For real-time PCR a Luna Script RT Super Mix Kit (New England Biolabs, Ipswich, MA, USA) was used. HPRT1 (Fwd: GCTTTCCTTGGTCAGGCAGTA, Rev: GTCTGGCTTATATCCAACACTTCGT, Probe: CAAGGTCGCAAGCTTGCTGGTGAAAAGGA), TATA-Box binding protein (TBP; Fwd: CACGAACCACGGCACTGATT, Rev: TTTTCTTGCTGCCAGTCTGGAC, Probe: TCTTCACTCTTGGCTCCTGTGCACA), and HMBS (TaqMan Gene Expression Assay from Thermo Fisher Scientific; Hs00609297_m1) were used as reference genes. The following Taqman gene expression assays were used: p300 (Hs00914223_m1), CBP (Hs00932878_m1), c-Myc (Hs00153408_m1), and Vimentin (Hs00185584_m1).

\section{Generation of doxycycline-inducible knockdown cell lines}

Stable cell lines with inducible p300 knockdown were generated by lentiviral-based transduction of shRNA vectors using the BLOCK-iT HiPerform Lentiviral Pol-II miR RNAi Expression System with emGFP from Invitrogen. Briefly, miR Select oligos (Hmi405238: shp300-1;
Hmi405239: shp300-2) were purchased from Life Technologies and ligated into pcDNA 6.2-GW/EmGFPmiR expression vector according to the manufacturer's protocol. Then the shRNAs were shuttled into the pDONR221 vector to generate entry clones. Entry plasmids together with a pENTR-tetOn (from pHR-TetCMV-eGFPdest ligated into pENTR 5'/CMVp vector) were then used in MultiSite Gateway reactions with pLenti6.4/R4R2/ V5-DEST to generate doxycycline-inducible shp300-1 and shp300-2. To generate cell lines stably expressing the doxycycline activator/repressor cassette, HEK293FT cells were first co-transfected with the packaging vectors pVSV-G and psPAX2 together with pHR-SFFV-rtTAM2T2A-Puro using X-tremeGENE reagent (Roche) according to the manufacturer's manual. Supernatants containing virus particles were collected $48 \mathrm{~h}$ after transfection, filtered through a $0.45 \mu \mathrm{m}$ membrane filter, and used for infection of target cells. Cells were selected with $2 \mu \mathrm{g} / \mathrm{mL}$ puromycin and subsequently transfected with packaging vectors together with the pLenti6.4 expression vector as described previously. Selection of infected cells was performed using $2 \mu \mathrm{g} / \mathrm{mL}$ blasticidine. For activation of the system, $100 \mathrm{ng} / \mathrm{mL}$ doxycycline was used. Activation status of the system was verified by GFP expression.

\section{Proliferation assay}

Cumulative population doubling levels (PDL) were determined by continuously seeding a defined number of cells in T25 flasks. Cell numbers were determined by CASY cell counter (Schärfe System, Reutlingen, Germany) every 3-4 days. Cumulative PDL was calculated with the following formula: $\mathrm{PDL}=3.32 \times(\log 10(\mathrm{X})-\log 10(\mathrm{Y}))+\mathrm{I}$, where $\mathrm{X}=$ number of cells at the end of growth period, $\mathrm{Y}=$ number of cells at the beginning of growth period, and $\mathrm{I}=$ initial population doubling level. To compare the growth curves, linear fit regression with the test for significance of slopes and intercepts was performed in GraphPad Prism 8.

\section{High-resolution colony formation assay}

Cell numbers were determined using CASY cell counter system (Schärfe System). Per $75 \mathrm{~cm}^{2}$ culture flask, 1000 cells were seeded and incubated for 10-14 days in the absence or presence of $100 \mathrm{ng} / \mathrm{mL}$ doxycycline. Cells were fixed with 100\% ice-cold methanol for 5 min and stained with crystal violet $(0.5 \%$ dissolved in PBS containing 20\% methanol (Sigma)) for $5 \mathrm{~min}$. Colony formation efficiency was determined by the software 
CATCH-colonies (https://catch-colonies.net). To correct for differences in the seeding density of the different stable shRNA cell lines, the colony formation efficiency was normalized to flasks that were seeded at the same time but not treated with doxycycline.

\section{Wound scratch assay}

Cells were seeded until they were nearly confluent in multiwell plates and treated with $100 \mathrm{ng} / \mathrm{mL}$ doxycycline or 10 HM CPI-637 (MedChem Express, Monmouth Junction, NJ, USA) for $96 \mathrm{~h}$ in total. After the first $24 \mathrm{~h}, 10 \mu \mathrm{M}$ of the proliferation inhibitor cytosine $\beta$-D-arabinofuranoside was added. After another $24 \mathrm{~h}$, a scratch was made using a 10- $\mu \mathrm{L}$ pipette tip. Images were taken after another $48 \mathrm{~h}$ and analyzed using the MRI Wound Healing Tool of ImageJ.

\section{Migration and invasion assay}

For migration and invasion assays, Boyden chamber inserts with $8 \mu \mathrm{m}$ pore size (Fluoroblok System, Becton Dickinson) were used. $3 \times 10^{4}$ cells per well of PC3-DR shCtrl, PC3-DR shp300-1, and PC3-DR shp300-2 were seeded in duplicate in serum-free medium. Bottom chambers were filled with medium containing 10\% FCS serving as chemoattractant. For invasion assays, inserts were coated with $30 \mu \mathrm{l}$ of matrigel (diluted 1:3 in serumfree medium; Corning). Cells were incubated for $48-72 \mathrm{~h}$ in the absence or presence of $100 \mathrm{ng} / \mathrm{mL}$ doxycycline or $20 \mu \mathrm{M}$ CPI-637, respectively. Afterwards, cells were stained with $2 \mu \mathrm{M}$ of calcein AM (Sigma) diluted in HBSS containing 1\% FCS. Fluorescent images were taken using a JuLI smart fluorescent cell analyzer (Science Services, Munich, Germany), and extinction/emission at 494/517 was measured using TECAN plate reader (Tecan Group Ltd., Männedorf, Switzerland).

\section{Immunofluorescence}

Cells were seeded on glass coverslips in the absence or presence of $100 \mathrm{ng} / \mathrm{mL}$ doxycycline for $72 \mathrm{~h}$. Cells were fixed with $4 \%$ paraformaldehyde and permeabilized with 1\% BSA in PBS containing 0.2\% Triton X-100. Washing steps were performed with 1\% BSA in PBS. AntiVimentin (1:500, Santa Cruz Biotechnology) was used as a primary antibody, together with the fluorescence-labeled secondary antibody goat anti-mouse 555 (ThermoFisher). Coverslips were mounted with Vectashield mounting medium containing DAPI.

\section{Viability assays}

PC3-DR cells were seeded in multi-well plates and treated with a range of CPI-637 concentrations. The solvent DMSO served as a control. The viability was measured using RealTime-Glo ${ }^{\text {TM }}$ MT Cell Viability Assay (Promega) after $72 \mathrm{~h}$ and the IC50 was calculated using a nonlinear fit model with variable slope of log transformed data in GraphPad Prism 8.

\section{Statistical analysis}

Statistical analysis was performed using GraphPad Prism 8 (GraphPad Software Inc.). Gaussian distribution of patient samples was determined using Kolmogorov-Smirnov test. Comparison of the two groups was performed using Student's $t$-test and Mann-Whitney $U$ test, depending on Gaussian distribution. Comparison of multiple groups was performed using one-way ANOVA and correcting for multiple testing using Bonferroni multiple comparison test. For integration of multiple independent replicates from methods that yield relative abundance values (qPCR, Western blot), each replicate was normalized to its average signal intensity. $P$-values of $<0.05$ were considered statistically significant and encoded in figure legends as follows: ${ }^{*} P<0.05 ;{ }^{* *} P<0.01 ;{ }^{* *} P<0.001$; and ${ }^{* * * *} P<0.0001$. If not stated otherwise, doxycycline-treated cells were normalized to non-treated control cells, and doxycycline-treated cells are shown in graphs.

\section{Results}

\section{p300 expression is increased in docetaxel-} treated patients

To evaluate whether p300 expression is altered upon docetaxel treatment, we analyzed tissue material of patients who received docetaxel by IHC staining using a specific p300 antibody (Supplementary Fig. 1A, B and $\mathrm{C}$, see section on supplementary materials given at the end of this article). In total, 28 patients were included, 14 of them received neoadjuvant docetaxel therapy before radical prostatectomy (RPE), while the other 14 patients did not receive chemotherapy prior to RPE. For detailed information about selected patients, please see the work of Puhr et al. (2012), where clinical data are fully described. We observed a significantly increased p300 expression in cancerous areas of docetaxel-treated patients compared to control patients (Fig. 1A and B), which was not the case in benign areas. Furthermore, p300 staining was observed mainly in nuclear areas.

This work is licensed under a Creative Commons Attribution 4.0 International License. 
Elevated p300 expression was further confirmed in publicly available datasets of mCRPC tissue samples from patients that suffered from relapse after docetaxel treatment (Kumar et al. 2016). Our analysis revealed significantly increased p300 levels (1.5-fold) in patients treated with docetaxel compared to patients that did not receive docetaxel at any time in their treatment course (Fig. 1C). To assess if docetaxel-mediated increase is specific for p300, we included the analysis of the closely related coactivator CBP. Interestingly, CBP expression levels were found slightly decreased in docetaxel-treated patients compared to non-docetaxel-treated patients (Fig. 1C). Furthermore, we analyzed AR expression and activity upon docetaxel treatment to evaluate whether the AR has any impact on docetaxel-mediated upregulation of $\mathrm{p} 300$. Of note, AR mRNA expression and androgen response were not significantly changed in patients that relapsed after docetaxel treatment compared to control patients (Supplementary Fig. 2A and B).

\section{Docetaxel-resistant and docetaxel-treated PCa cells show increased p300 expression}

Next, we compared p300 expression in docetaxelresistant (DR) derivatives of commonly employed PCa cell lines (PC3-DR, DU145-DR, and CWR22RV1-DR) relative to their docetaxel-sensitive counterparts. p300 protein levels were significantly increased (1.5-fold) in all three docetaxel-resistant cell lines tested compared to docetaxel-sensitive counterparts (Fig. 2A), whereas mRNA levels were unchanged (Supplementary Fig. 3A). Increased p300 protein expression in docetaxel-resistant PC3-DR and DU145-DR was confirmed by IHC staining (Fig. 2B). CBP mRNA and protein expression were not significantly regulated in docetaxel-resistant cells compared to sensitive counterparts (except downregulated CBP mRNA expression in PC3-DR cells, Supplementary Fig. 3B and C).

To understand the molecular basis of these findings, we analyzed p300 expression upon short-term docetaxel treatment in several PCa cell lines. To this end, we measured p300 mRNA and protein expression in PC3, DU145, and CWR22RV1 cells after treatment with docetaxel for $72 \mathrm{~h}$. Protein expression of p300 significantly increased (2-2.5-fold increase) in all three cell lines upon docetaxel treatment (Fig. 2C), whereas mRNA levels were not significantly changed (Supplementary Fig. 4A). Time-course experiments revealed that $\mathrm{p} 300$ protein, but not mRNA expression, increased within $8 \mathrm{~h}$ of docetaxel treatment and reached a plateau after $16 \mathrm{~h}$ (Supplementary Fig. 4B and C). Interestingly, p300 expression in docetaxelresistant cells decreased if the cells were cultured without docetaxel (Supplementary Fig. 4D and E). To investigate
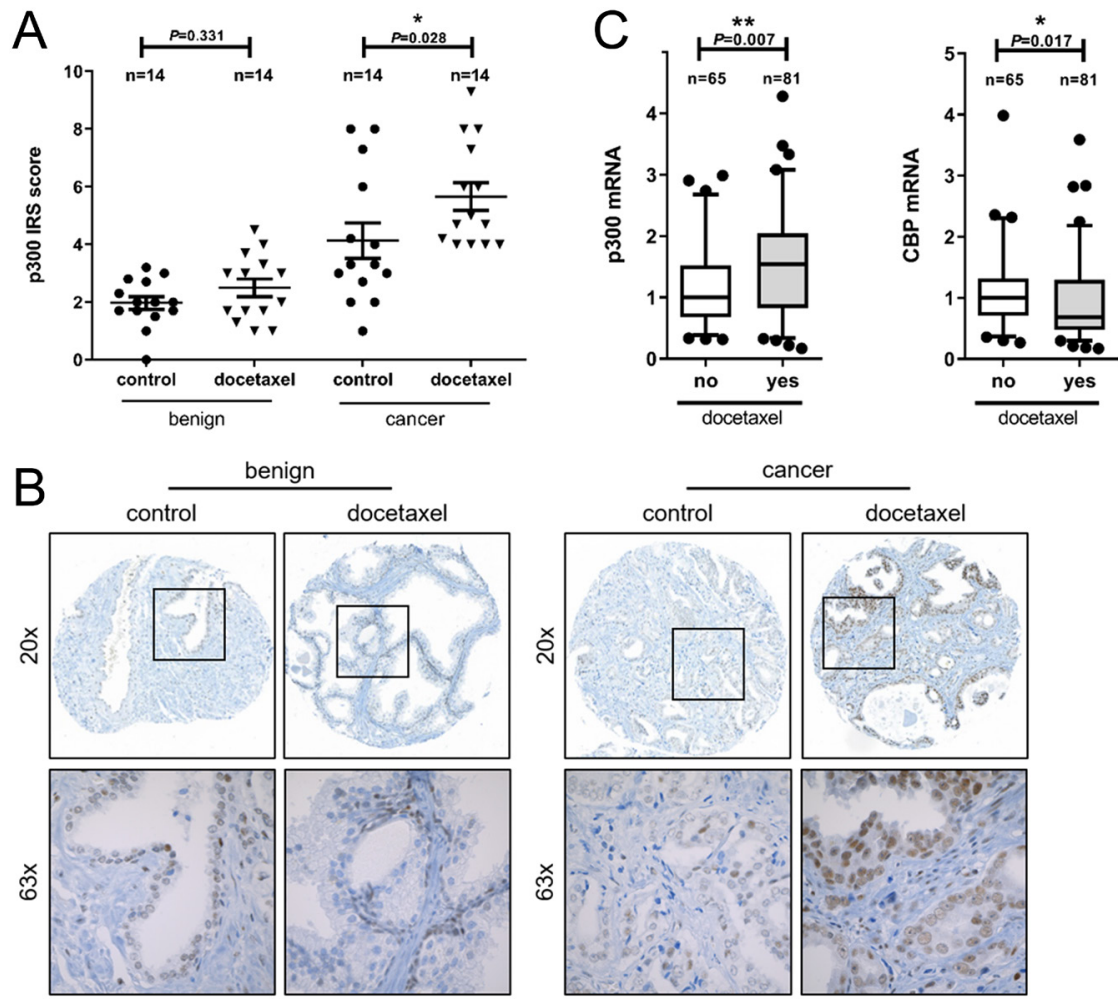

Figure 1

Expression of p300 is increased upon docetaxel treatment. (A) Quantification of p300 immunoreactivity scores (IRS) after IHC staining (Mann-Whitney $U$ test; scatter dot blot with line at mean + S.E.M.). (B) IHC staining of p300 (nuclear localization). Upregulation of p300 expression in cancerous tissue of docetaxel-treated patients compared to control patients. (C) p300 and CBP mRNA expression were determined in samples of docetaxel-treated and docetaxel-untreated mCRPC patients (Mann-Whitney $U$ test; box whisker plot with 5-95 percentile). https://erc.bioscientifica.com https://doi.org/10.1530/ERC-19-0488 (c) 2020 The authors Published by Bioscientifica Ltd. Printed in Great Britain
This work is licensed under a Creative Commons Attribution 4.0 International License. 
A

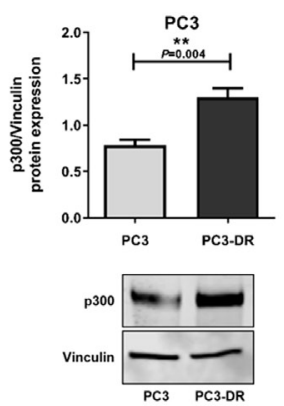

B

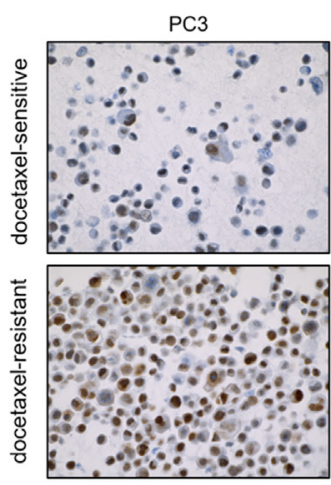

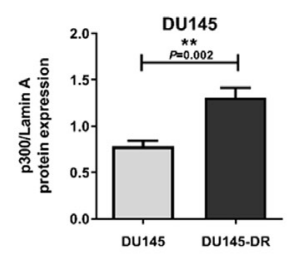
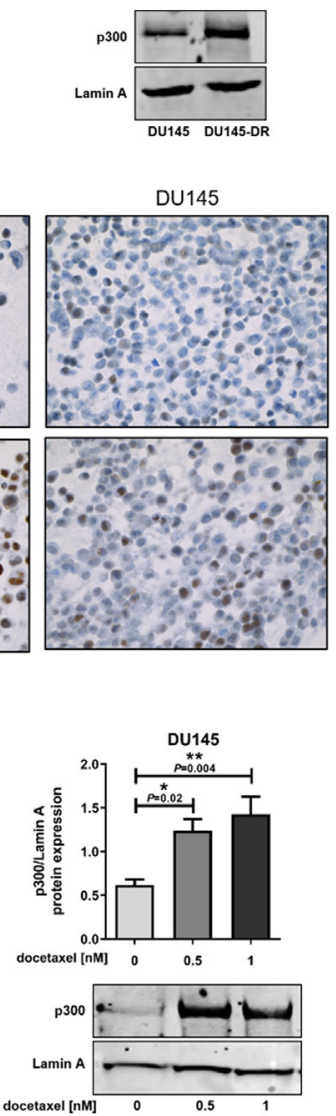
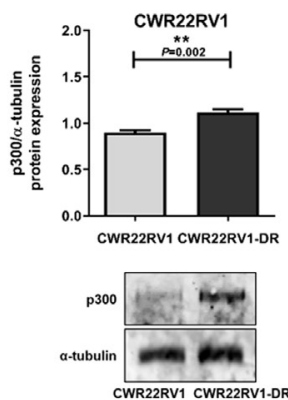

CWR22RV1
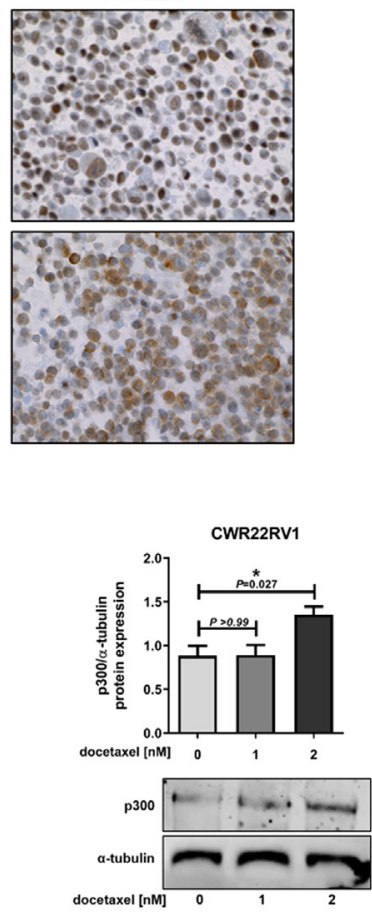

Figure 2

p300 expression is increased in docetaxelresistant prostate cancer cells. (A) Comparison of p300 protein expression between docetaxelsensitive and docetaxel-resistant (DR) PC3 $(n=4)$, DU145 $(n=5)$, and CWR22RV1 $(n=4)$. Data represent mean + S.E.M. (t-test). (B) IHC staining for p300 in docetaxel-resistant PC3-DR, DU145-DR, and CWR22RV1-DR compared to docetaxelsensitive counterparts. Magnification 40×. (C) PC3 $(n=5), \operatorname{DU} 145(n=5)$, and CWR22RV1 $(n=4)$ were treated with the indicated concentrations of docetaxel for $72 \mathrm{~h}$, and p300 protein expression was analyzed by Western blot, and one representative Western blot is shown. Values represent mean + S.E.M. (one-way ANOVA). whether docetaxel influences the protein degradation rate of p300, translation was blocked with cycloheximide (CHX). Of note, p300 had a protein half-life time of around $6 \mathrm{~h}$ in untreated PC3 cells, whereas addition of docetaxel (PC3 and PC3-DR) stabilized the protein (Supplementary Fig. 4F).

We also analyzed the expression levels of the welldescribed p300 downstream target c-Myc, which is a known oncogene in PCa (Koh et al. 2010). Our analysis revealed no significant change of c-Myc mRNA levels (Supplementary Fig. 5A), but a Myc gene expression activity score was significantly increased in docetaxel-treated patients (Supplementary Fig. 5B). Additionally, docetaxel-resistant PC3-DR and DU145-DR showed higher c-Myc protein expression compared to docetaxel-sensitive counterparts (Supplementary Fig. 5C), and c-Myc protein expression was increased (1.5-fold) in DU145 upon treatment with $1 \mathrm{nM}$ docetaxel compared to the control (Supplementary Fig. 5D).

\section{Docetaxel-resistant cells show reduced colony formation ability upon p300 inhibition}

To study a possible mechanistic role of p300 in docetaxel resistance, PC3, CWR22RV1, and their docetaxel-resistant counterparts were stably transduced with doxycyclineinducible short hairpin (sh)p300 vectors. We selected these cell lines to include both AR-negative (PC3 and PC3-DR) as well as AR-positive (CWR22RV1 and CWR22RV1-DR) sublines. Doxycycline treatment activated the inducible system as indicated by homogeneous expression of vector-integrated GFP (Fig. 3A), which led to decreased p300 protein expression (Fig. 3B) and activity by decreased acetylation of histone h3 on lysine 18 (Fig. 3C). Of note, p300 knockdown had no biologically consistent effect on the proliferation rate of docetaxelsensitive or docetaxel-resistant cells over a period of 20 days (Fig. 3D). The significantly reduced proliferation https://erc.bioscientifica.com https://doi.org/10.1530/ERC-19-0488
(C) 2020 The authors Published by Bioscientifica Ltd. Printed in Great Britain
This work is licensed under a Creative Commons Attribution 4.0 International License. 
rate of PC3-DR shp300-2 was not reproducible with the shp300-1 construct and is, thus, likely an artifact or off-target effect. To exclude the possibility that CBP is upregulated by p300 inhibition, thus compensating for the effects of p300 downregulation, we analyzed CBP expression upon p300 knockdown. As expected, specific downregulation of p300 had no impact on CBP expression (Supplementary Fig. 6).

An important characteristic of aggressive tumor cells is the ability to form colonies, thereby assessing a single cell's ability to undergo unlimited division. In docetaxel-sensitive PC3 and CWR22RV1, knockdown of p300 had no significant effect on the clonogenic potential (Fig. 4A). However, interestingly, p300 inhibition in docetaxel-resistant PC3-DR and CWR22RV1-DR significantly reduced colony formation efficiency (reduction by 40-50\% in PC3-DR and 20-30\% in CWR22RV1-DR; Fig. 4B).

\section{Cell migration and invasion are impaired upon p300 downregulation in docetaxel-resistant cells}

It has previously been shown that p300 is involved in migration and invasion in PCa cells (Santer et al. 2011). Therefore, we wanted to test if p300 inhibition might also impair cell migration and invasion in docetaxel-resistant cells. We employed PC3-DR cells for these experiments since the migration ability of CWR22RV1-DR cells is low. We first performed wound scratch assays that revealed a 2.5-fold decrease in the wound healing rate upon p300 downregulation (Fig. 5A). Consistently, significantly decreased cell migration by $~ 50 \%$ was observed in Boyden chambers following p300 downregulation (Fig. 5B). Moreover, invasion assays, which were conducted on matrigel-coated membranes, also showed a significantly reduced invasion ability (reduction by 50\%) upon p300 downregulation (Fig. 5C). These findings were confirmed
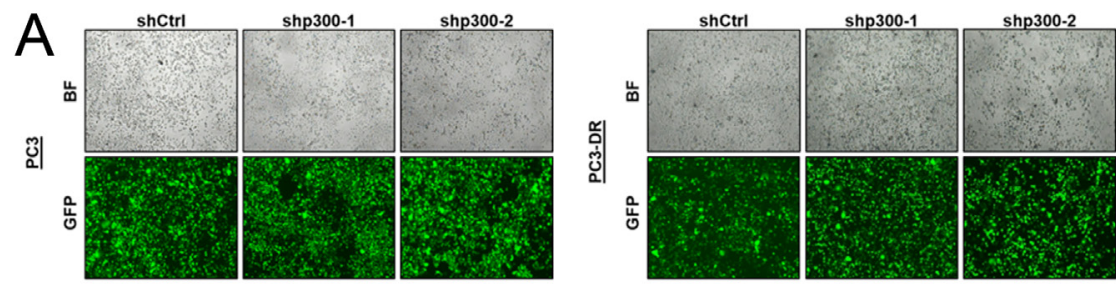

B
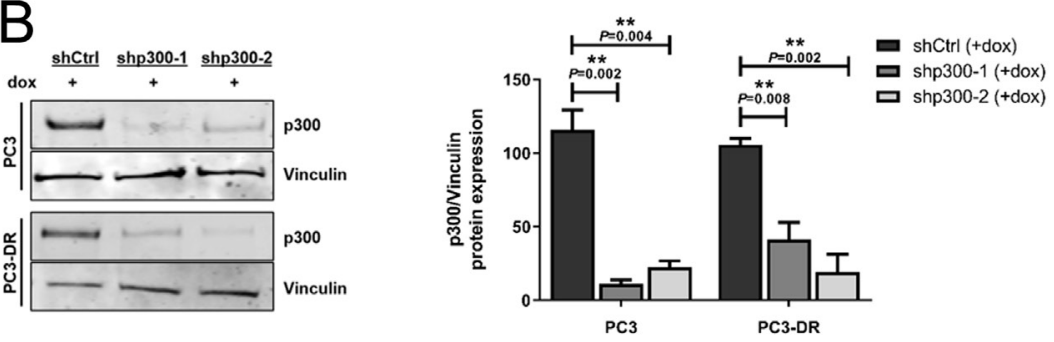

C

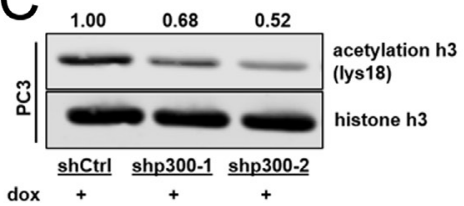

$\mathrm{D}$

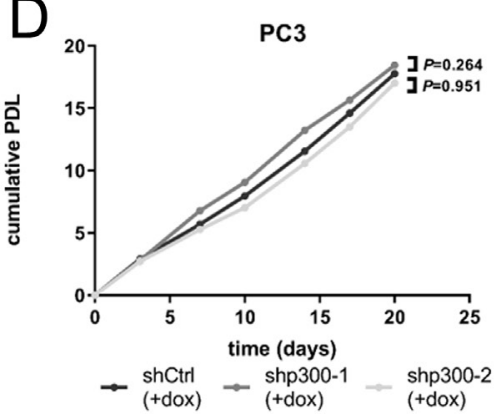

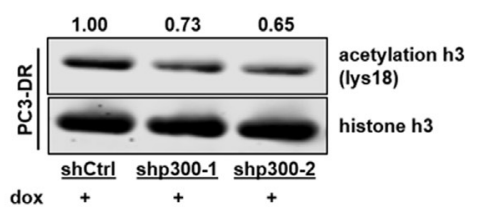

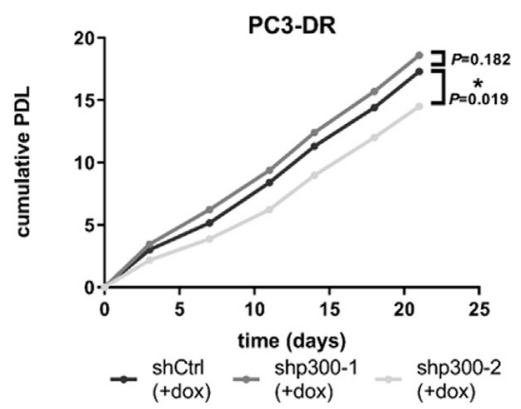

Figure 3

Establishment and validation of doxycyclineinducible p300 knockdown cell lines using a non-targeting control (shCtrl) sequence and two specific p300-shRNA sequences (shp300-1 and shp300-2). Docetaxel-sensitive PC3 and docetaxel-resistant PC3-DR are shown here, representing all mentioned cell lines. For better visualization in the following experiments, only doxycycline-treated shCtrl, shp300-1, and shp300-2 replicates are shown. Confirmation of (A) uniform expression of shRNA-constructs by fluorescence microscopy ( $\mathrm{BF}=$ bright field, GFP = green fluorescent protein) and magnification 40x and (B) p300 knockdown by Western blot analysis after activation of shp300-sequences with $100 \mathrm{ng} / \mathrm{mL}$ doxycycline for $72 \mathrm{~h}$. Values indicated are mean + S.E.M. (one-way ANOVA, $n=3$ ). (C) Decreased p300 activity was confirmed by analysis of histone h3 acetylation on lysine18 in PC3 and PC3-DR cells. One representative Western blot out of three independent experiments is shown. (D) Cumulative population doubling levels (PDL, $n=1$ ) of PC3 and PC3-DR cells over time with downregulated $\mathrm{p} 300$ were calculated by cell number measurement upon each passage (linear fit regression and test for significance of slopes and intercepts). https://erc.bioscientifica.com https://doi.org/10.1530/ERC-19-0488 Printed in Great Britain
This work is licensed under a Creative Commons Attribution 4.0 International License. 

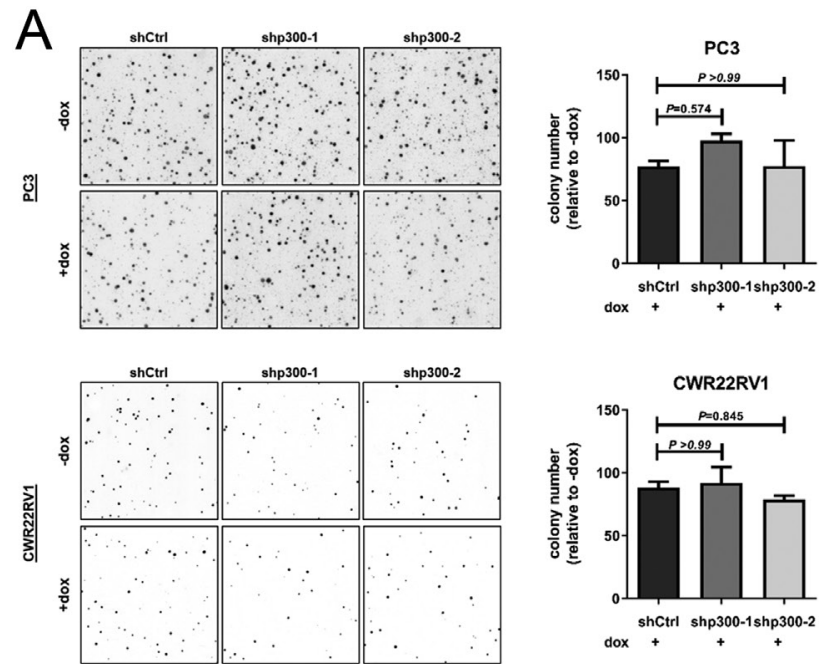
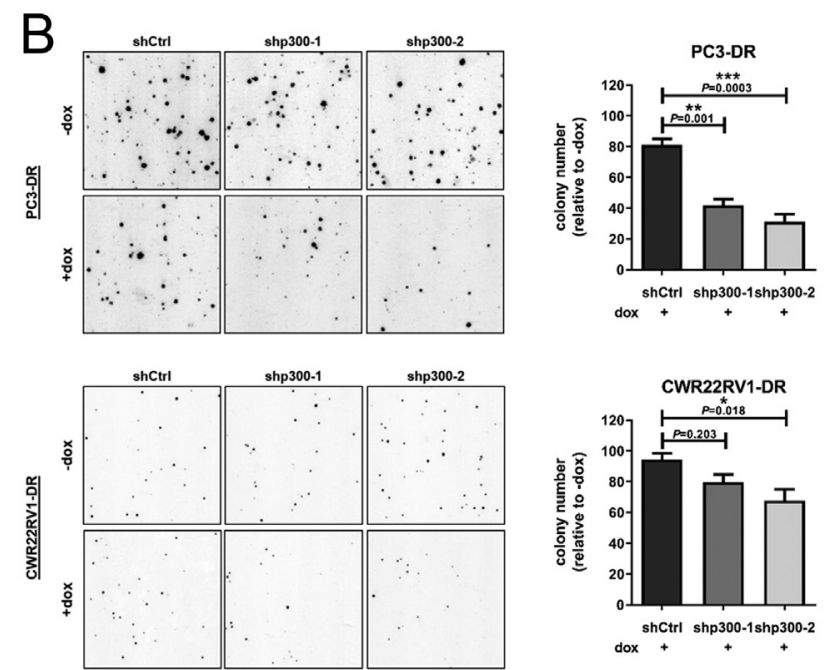

Figure 4

p300 inhibition decreases colony formation ability of docetaxel-resistant cells. Representative images and quantification of high-resolution colony formation assays of docetaxel-sensitive PC3 and CWR22RV1 (A) and docetaxel-resistant PC3-DR and CWR22RV1-DR (B). Colony numbers were analyzed by the software CATCH-colonies. Data represent mean + S.E.M. (one-way ANOVA, $n=3$ ).

by the use of the dual inhibitor of p300 and CBP CPI637. Viability assays were performed to calculate the IC50 for PC3-DR cells (Supplementary Fig. 7). It was determined that IC50 for CPI-637 is $17.52 \mu \mathrm{M}$. PC3-DR cells treated with CPI-637 for $72 \mathrm{~h}$ revealed a significantly decreased migration and invasion ability (reduction by $50-60 \%$, Fig. 5D, E and F). Vimentin is a cytoskeleton component that plays an important role in migration and was therefore analyzed in p300-silenced PC3-DR cells by immunofluorescence, where we observed a significantly decreased vimentin protein expression (by 60-70\%, Fig. 5G). Reduced vimentin levels were additionally confirmed by qRT-PCR and Western blot analysis (Fig. 5H).

\section{Discussion}

Although chemotherapy for CRPC patients confers a clinical benefit for patients, there is no curative treatment available for late stages of PCa. Development of chemotherapy resistance occurs rapidly, and it remains largely unclear which factors are differentially expressed during chemotherapy and might contribute to docetaxel insensitivity. Thus, it is important to understand the molecular mechanisms and to find new therapeutic targets to overcome therapy failure.

As a coactivator of the AR, p300 is involved in many biological processes such as differentiation, proliferation, and cell cycle regulation (Iyer et al. 2004) and has already been associated with tumor progression and poor prognosis
(Debes et al. 2003). Furthermore, p300 expression is increased upon androgen deprivation (Heemers et al. 2007) and plays an essential role in ligand-independent transactivation of the AR in androgen-independent PCa cells (Debes et al. 2002).

The main finding of this study is that p300 is upregulated upon docetaxel treatment in primary PCa and mCRPC tissue samples as well as in docetaxel-sensitive and docetaxel-resistant PCa cells. IHC staining was performed in samples of patients who received neoadjuvant docetaxel therapy at the Department of Urology of the Medical University of Innsbruck. Of course, these samples do not reflect the same clinical stage as docetaxel-resistant cells. However, patients who receive chemotherapy for PCa in the Authors' institution are not subjected to removal of tumor tissue. Therefore, the samples from individuals who received neoadjuvant docetaxel therapy were selected to be able to analyze p300 expression in patient material. Furthermore, metastatic tissue samples obtained by rapid autopsy from a publicly available transcriptome dataset including $\mathrm{mCRPC}$ patients that received docetaxel in their treatment course were analyzed. Increased 300 expression was observed in vitro in docetaxel-resistant AR-positive and AR-negative cells (PC3-DR and CWR22RV1-DR) compared to their respective docetaxel-sensitive counterparts. This finding indicates that p300 is upregulated by docetaxel regardless of AR expression and is consistent with previous publications documenting multiple functions of p300 in PCa also independently of the AR (Debes et al. 2003, Santer et al. 2011). This is also in concordance with the 
A

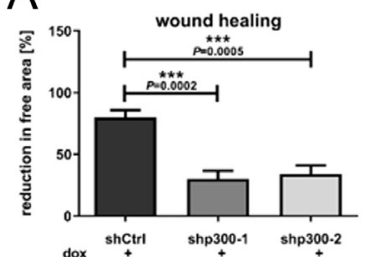

dox shctrl shp300-1 shp300-2

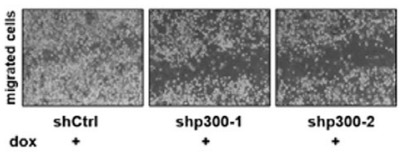

D

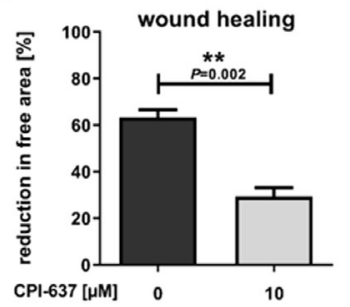

B
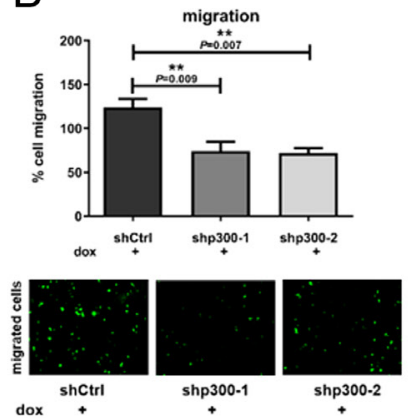

E

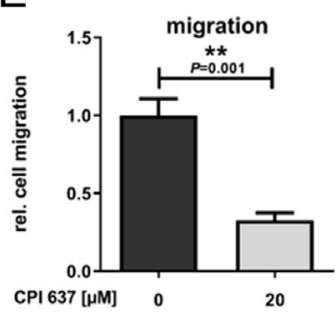

C

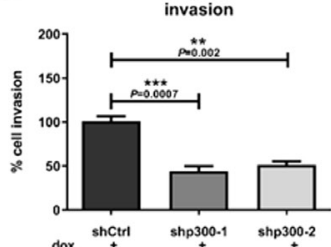

dox $\stackrel{\text { shctrl }}{+} \underset{+}{\operatorname{shp} 300.1}$ shp $300.2_{+}$

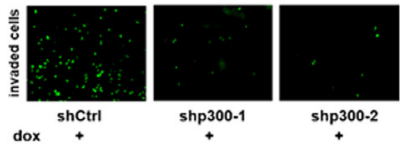

F

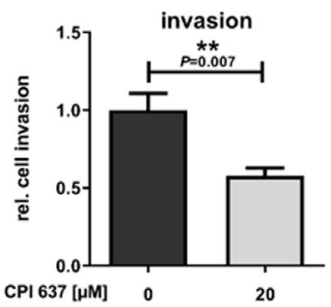

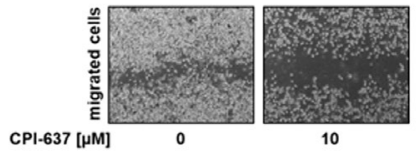

CPI-637 [MM]

10

G

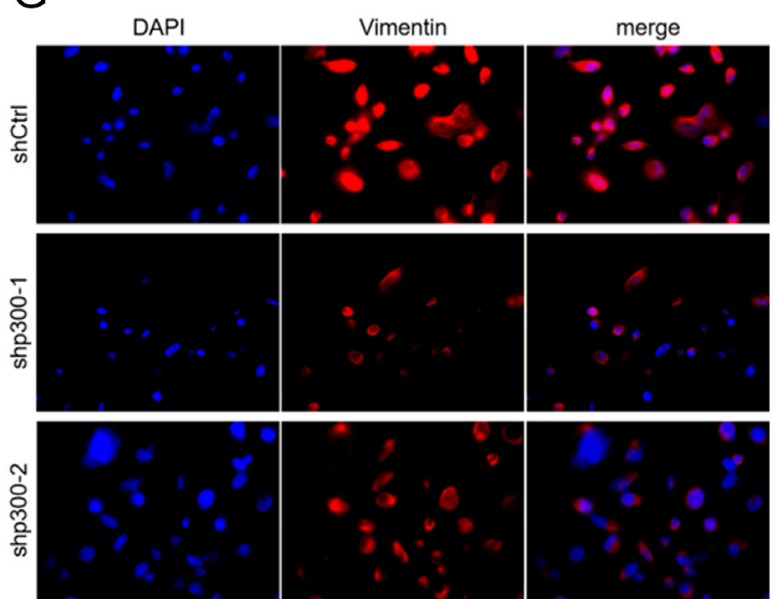

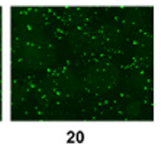

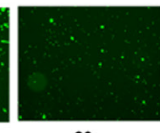

20

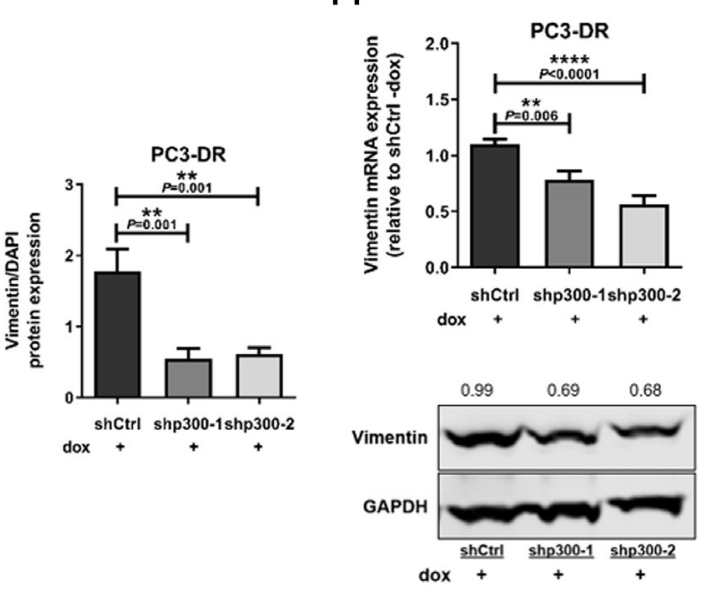

$\mathrm{H}$

Figure 5

p300 downregulation decreases cell migration and invasion. (A) Wound scratch assays were performed on confluent layers of PC3-DR shCtrl, shp300-1, and shp300-2 treated with $100 \mathrm{ng} / \mathrm{mL}$ doxycycline. Images were taken $48 \mathrm{~h}$ after scratch and analyzed by Image $(n=6)$. (B) PC3-DR were seeded in Boyden chambers and shp300 sequences were activated with $100 \mathrm{ng} / \mathrm{mL}$ doxycycline for $72 \mathrm{~h}$. Cell migration was measured after staining with calcein and visualized by fluorescence microscopy $(n=4)$. (C) Invasion assays were conducted as in B, except that Boyden chambers were pre-coated with Matrigel $(n=3)$. Values indicated in A-C denote mean + s.E.M. (one-way ANOVA). (D) Wound scratch assays on PC3-DR treated with $10 \mu \mathrm{M}$ CPI-637. Data represent mean + S.E.M. ( $($-Test, $n=3)$. (E) Migration $(n=4)$ and (F) invasion assays $(n=5)$ of PC3-DR treated with $20 \mu \mathrm{M}$ CPI-637. Values indicated are mean + S.E.M. (t-test). (G) Immunofluorescence staining for vimentin (red) and quantification relative to counterstaining of nuclei (blue). Original magnification 630×. (H) mRNA (qPCR) and protein expression (Western blot) of vimentin. Data in G-H represent mean + S.E.M. (one-way ANOVA, $n=5$ ).

finding that AR expression and activity are not changed upon docetaxel treatment; although, in previous studies it has been shown that docetaxel impairs transcriptional activity of the AR (Zhu et al. 2010). Other groups reported an inhibitory effect of docetaxel on AR activity by interfering with AR intracellular trafficking https://erc.bioscientifica.com https://doi.org/10.1530/ERC-19-0488 (c) 2020 The authors Published by Bioscientifica Ltd. Printed in Great Britain
This work is licensed under a Creative Commons Attribution 4.0 International License. 
(Martin et al. 2015) and nuclear translocation (ThadaniMulero et al. 2014).

Contrary to p300, the closely related coactivator CBP was not upregulated upon docetaxel treatment. Thus, we conclude that docetaxel-induced upregulation is specific for p300. Hatano and colleagues reported a role of c-Myc, which is a known downstream target of p300 in docetaxel resistance (Hatano et al. 2013). Concordant with their study, we observed an increased expression of c-Myc in docetaxel-treated patients; however, the upregulation was not statistically significant. Nevertheless, Myc activity was significantly increased in patients who received docetaxel. Ogiwara and colleagues also reported that p300 ablation caused downregulation of Myc expression in CBPdeficient cells and thereby suppressed cancer cell growth (Ogiwara et al. 2016). We assume that p300 upregulation by docetaxel is not mediated by c-Myc, but c-Myc is affected in consequence as a target gene of p300.

We found that p300 mRNA expression was not significantly changed upon docetaxel treatment, which indicates that p300 protein increase is not due to increased transcriptional activity. p300 protein expression also does not increase because of elevated translation, since p300 protein expression in docetaxel-resistant cells was unchanged upon translation inhibition, which suggests that p300 is protected from proteasomal degradation. In a previous study, it was found that androgens downregulate p300 protein but not mRNA expression (Heemers et al. 2007). Those results suggest similarities in regulation of p300 by docetaxel and androgens.

It has been previously shown that the competitive histone acetyltransferase p300/CBP inhibitor C646 reduced colony formation in AML cell lines and primary blasts (Gao et al. 2013). While this highlights the capacities of HAT inhibitors, there are issues with the potency and selectivity of these early inhibitors (Lasko et al. 2017). Meanwhile, more effective bromodomain and extra-terminal (BET) inhibitors that prevent protein-protein interactions between BET proteins and acetylated histones have been developed. The BET inhibitors INCB054329 and INCB057643 have been shown to be effective as single agents in PCa. Likely, novel BET inhibitors will be combined with existing therapies, in particular, for therapy-resistant PCa (Vazquez et al. 2019). In this study, we initially employed a shRNA approach to down-regulate p300 and exclude non-specific effects. We confirmed effects of shRNA-mediated p300 downregulation on migration ability of docetaxel-resistant cells with the bromodomain inhibitor CPI-637 that is specific for p300 and CBP, where we observed similar results as with genetic p300 downregulation.
In contrast to docetaxel-sensitive cells, p300 inhibition in docetaxel-resistant PC3-DR and CWR22RV1-DR significantly reduced colony formation efficiency. Downregulation of p300 had no specific effects on proliferation of docetaxel-resistant cells, indicating that p300 inhibition indeed impaired the colony-initiating capacity and that the reduced colony number is not just a secondary effect. It has already been described that docetaxel-resistant cells show a stem-cell-like phenotype (Puhr et al. 2012, Marin-Aguilera et al. 2014), suggesting that p300 inhibition is effective in conditions in which pathways connected to colony formation and tumorinitiation play a central role. A possible explanation as to why p300 inhibition shows no effects on colony formation of docetaxel-sensitive cells could be the formation of p300complexes. It has been described that the cysteine protease USP24 stabilizes p300 and thereby increases acetylation of histone h3 (Wang et al. 2018). The CtBP1-p300-FOXO 3a complex was found to repress apoptotic regulators Bax and Bim in osteosarcoma cells (Li et al. 2019). Cell proliferation is regulated by p300 in complex with the transcriptional repressor YY1 and HDAC2 (Tang et al. 2019). Future studies of therapy resistance in PCa should therefore examine and quantitate the complexes relevant to $\mathrm{p} 300$. In contrast to $\mathrm{PCa}$, in some models of breast cancer, p300 is considered a tumor inhibitor (Asaduzzaman et al. 2017). However, the histone methyltransferase DOT1L in complex with p300 and c-Myc enhanced cellular stemness (Cho et al. 2015). The reasons for obviously contrasting effects of p300 on stemness in breast cancer are not yet known.

p300 inhibition significantly decreased cell migration and invasion in PC3-DR cells and reduced vimentin expression. These findings indicate that p300 is involved in cellular pathways that regulate migration and invasion in PCa and are in concordance with previous studies, where they reported involvement of p300 in migration in nasopharyngeal carcinoma and breast cancer cells (Fermento et al. 2014, Liao et al. 2017).

Taken together, p300 could be a valid target in docetaxel-resistant $\mathrm{PCa}$ as it reduces the metastatic potential of docetaxel-resistant cells by reducing colony formation, migration, and invasion capability. So far, multiple factors that contribute to the development of docetaxel resistance in PCa have been described (Patterson et al. 2006, Deng et al. 2019, Kapur et al. 2019). Therefore, docetaxel resistance in $\mathrm{PCa}$ is heterogeneous, thus suggesting that medical intervention may be based on a personalized approach. This issue is particularly relevant because p300 inhibitors enter clinical trials in oncology, thus pointing to appropriate selection of patients who

This work is licensed under a Creative Commons Attribution 4.0 International License. ed from Bioscientifica.com at 04/26/2023 09:57:30AM 
will benefit from specific p300 targeting in combinations with other drugs approved or tested for treatment of PCa.

\section{Supplementary materials}

This is linked to the online version of the paper at https://doi.org/10.1530/ ERC-19-0488.

\section{Declaration of interest}

The authors declare that there is no conflict of interest that could be perceived as prejudicing the impartiality of the research reported.

\section{Funding}

This work was supported by the Austrian Science Fund FWF grant W1101-B12 to Z Culig and P31122 to N Sampson.

\section{Author contribution statement}

M Gruber and L Ferrone performed experiments. M Gruber analyzed data and wrote the first version of the manuscript. M Puhr organized and supervised immunohistochemical analyses and supervised in vitro experiments with chemoresistance models. $T$ Furlan performed experiments with the bromodomain inhibitor. F R Santer, T Furlan, I Eder, and N Sampson provided experimental suggestions and analyzed data. M Puhr and G Schäfer established TMA. F Handle established the experimental system, performed the bioinformatic analysis of the transcriptome dataset, and supervised in vitro experiments. Z Culig was responsible for conception and design of the study, data analysis, and supervision. All authors contributed to manuscript writing.

\section{Acknowledgements}

The authors thank Sarah Peer for immunohistochemical staining; Eberhard Steiner, M.Sc., for patient selection and statistical analysis; Prof Walther Parson for cell line authentication; Prof Dr. William Watson for providing CWR22RV1 and CWR22RV1-DR cells; Dr Martin Hermann for help with immunofluorescence staining; Prof Helmut Klocker and $\mathrm{Dr}$ Maximilian Brandt for proofreading the manuscript, and all members of the Division of Experimental Urology at the Medical University of Innsbruck for helpful discussions. F Handle and Z Culig, the joint senior authors.

\section{References}

Asaduzzaman M, Constantinou S, Min H, Gallon J, Lin ML, Singh P, Raguz S, Ali S, Shousha S, Coombes RC, et al. 2017 Tumour suppressor EP300, a modulator of paclitaxel resistance and stemness, is downregulated in metaplastic breast cancer. Breast Cancer Research and Treatment 163 461-474. (https://doi.org/10.1007/s10549-0174202-z)

Chan HM \& La Thangue NB 2001 p300/CBP proteins: HATs for transcriptional bridges and scaffolds. Journal of Cell Science $\mathbf{1 1 4}$ 2363-2373.

Cho MH, Park JH, Choi HJ, Park MK, Won HY, Park YJ, Lee CH, Oh SH, Song YS, Kim HS, et al. 2015 DOT1L cooperates with the c-Myc-p300 complex to epigenetically derepress CDH1 transcription factors in breast cancer progression. Nature Communications 6 7821. (https:// doi.org/10.1038/ncomms8821)
Comuzzi B, Nemes C, Schmidt S, Jasarevic Z, Lodde M, Pycha A, Bartsch G, Offner F, Culig Z \& Hobisch A 2004 The androgen receptor co-activator CBP is up-regulated following androgen withdrawal and is highly expressed in advanced prostate cancer. Journal of Pathology 204 159-166. (https://doi.org/10.1002/path.1609)

Debes JD, Schmidt LJ, Huang H \& Tindall DJ 2002 p300 mediates androgen-independent transactivation of the androgen receptor by interleukin 6. Cancer Research 62 5632-5636.

Debes JD, Sebo TJ, Lohse CM, Murphy LM, Haugen DA \& Tindall DJ 2003 p300 in prostate cancer proliferation and progression. Cancer Research 63 7638-7640.

Deng L, Gu X, Zeng T, Xu F, Dong Z, Liu C \& Chao H 2019 Identification and characterization of biomarkers and their functions for docetaxel-resistant prostate cancer cells. Oncology Letters 18 3236-3248. (https://doi.org/10.3892/ol.2019.10623)

Fermento ME, Gandini NA, Salomon DG, Ferronato MJ, Vitale CA, Arevalo J, Lopez Romero A, Nunez M, Jung M, Facchinetti MM, et al. 2014 Inhibition of p300 suppresses growth of breast cancer. Role of p300 subcellular localization. Experimental and Molecular Pathology 97 411-424. (https://doi.org/10.1016/j.yexmp.2014.09.019)

Gao XN, Lin J, Ning QY, Gao L, Yao YS, Zhou JH, Li YH, Wang LL \& Yu L 2013 A histone acetyltransferase p300 inhibitor C646 induces cell cycle arrest and apoptosis selectively in AML1-ETO-positive AML cells. PLOS ONE 8 e55481. (https://doi.org/10.1371/journal. pone.0055481)

Hanzelmann S, Castelo R \& Guinney J 2013 GSVA: gene set variation analysis for microarray and RNA-seq data. BMC Bioinformatics 147. (https://doi.org/10.1186/1471-2105-14-7)

Hatano K, Yamaguchi S, Nimura K, Murakami K, Nagahara A, Fujita K, Uemura M, Nakai Y, Tsuchiya M, Nakayama M, et al. 2013 Residual prostate cancer cells after docetaxel therapy increase the tumorigenic potential via constitutive signaling of CXCR4, ERK1/2 and c-Myc. Molecular Cancer Research 11 1088-1100. (https://doi. org/10.1158/1541-7786.MCR-13-0029-T)

Heemers HV, Sebo TJ, Debes JD, Regan KM, Raclaw KA, Murphy LM, Hobisch A, Culig Z \& Tindall DJ 2007 Androgen deprivation increases p300 expression in prostate cancer cells. Cancer Research $\mathbf{6 7}$ 3422-3430. (https://doi.org/10.1158/0008-5472.CAN-06-2836)

Iyer NG, Ozdag H \& Caldas C 2004 p300/CBP and cancer. Oncogene 23 4225-4231. (https://doi.org/10.1038/sj.onc.1207118)

James ND, Sydes MR, Clarke NW, Mason MD, Dearnaley DP, Spears MR, Ritchie AW, Parker CC, Russell JM, Attard G, et al. 2016 Addition of docetaxel, zoledronic acid, or both to first-line long-term hormone therapy in prostate cancer (STAMPEDE): survival results from an adaptive, multiarm, multistage, platform randomised controlled trial. Lancet 387 1163-1177. (https://doi.org/10.1016/S01406736(15)01037-5)

Kalkhoven E 2004 CBP and p300: HATs for different occasions. Biochemical Pharmacology 68 1145-1155. (https://doi.org/10.1016/j. bcp.2004.03.045)

Kapur N, Mir H, Sonpavde GP, Jain S, Bae S, Lillard JW. \& Singh S 2019 Prostate cancer cells hyper-activate CXCR6 signaling by cleaving CXCL16 to overcome effect of docetaxel. Cancer Letters 454 1-13. (https://doi.org/10.1016/j.canlet.2019.04.001)

Koh CM, Bieberich CJ, Dang CV, Nelson WG, Yegnasubramanian S \& De Marzo AM 2010 MYC and prostate cancer. Genes and Cancer 1 617-628. (https://doi.org/10.1177/1947601910379132)

Kumar A, Coleman I, Morrissey C, Zhang X, True LD, Gulati R, Etzioni R, Bolouri H, Montgomery B, White T, et al. 2016 Substantial interindividual and limited intraindividual genomic diversity among tumors from men with metastatic prostate cancer. Nature Medicine 22 369-378. (https://doi.org/10.1038/nm.4053)

Kyriakopoulos CE, Chen YH, Carducci MA, Liu G, Jarrard DF, Hahn NM, Shevrin DH, Dreicer R, Hussain M, Eisenberger M, et al. 2018 Chemohormonal therapy in metastatic hormone-sensitive prostate cancer: long-term survival analysis of the randomized phase III

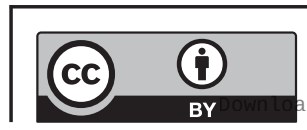

This work is licensed under a Creative Commons Attribution 4.0 International License. 
E3805 CHAARTED trial. Journal of Clinical Oncology 36 1080-1087. (https://doi.org/10.1200/JCO.2017.75.3657)

Lasko LM, Jakob CG, Edalji RP, Qiu W, Montgomery D, Digiammarino EL, Hansen TM, Risi RM, Frey R, Manaves V, et al. 2017 Discovery of a selective catalytic p300/CBP inhibitor that targets lineage-specific tumours. Nature 550 128-132. (https://doi. org/10.1038/nature24028)

Lavaud P, Gravis G, Foulon S, Joly F, Oudard S, Priou F, Latorzeff I, Mourey L, Soulie M, Delva R, et al. 2018 Anticancer activity and tolerance of treatments received beyond progression in men treated upfront with androgen deprivation therapy with or without docetaxel for metastatic castration-naive prostate cancer in the GETUG-AFU 15 phase 3 trial. European Urology 73 696-703. (https:// doi.org/10.1016/j.eururo.2017.09.022)

Li C, Xiao XQ, Qian YH \& Zhou ZY 2019 The CtBP1-p300-FOXO3a transcriptional complex represses the expression of the apoptotic regulators Bax and Bim in human osteosarcoma cells. Journal of Cellular Physiology 234 22365-22377. (https://doi.org/10.1002/jcp.28802)

Liao ZW, Zhao L, Cai MY, Xi M, He LR, Yu F, Zhou TC \& Liu MZ 2017 P300 promotes migration, invasion and epithelial-mesenchymal transition in a nasopharyngeal carcinoma cell line. Oncology Letters 13 763-769. (https://doi.org/10.3892/ol.2016.5491)

Marin-Aguilera M, Codony-Servat J, Reig Ò, Lozano JJ, Fernandez PL, Pereira MV, Jimenez N, Donovan M, Puig P, Mengual L, et al. 2014 Epithelial-to-mesenchymal transition mediates docetaxel resistance and high risk of relapse in prostate cancer. Molecular Cancer Therapeutics 13 1270-1284. (https://doi.org/10.1158/1535-7163.MCT13-0775)

Martin SK, Banuelos CA, Sadar MD \& Kyprianou N 2015 N-terminal targeting of androgen receptor variant enhances response of castration resistant prostate cancer to taxane chemotherapy. Molecular Oncology 8 628-639. (https://doi.org/10.1016/j. molonc.2014.10.014)

Ogiwara H, Sasaki M, Mitachi T, Oike T, Higuchi S, Tominaga Y \& Kohno T 2016 Targeting p300 addiction in CBP-deficient cancers causes synthetic lethality by apoptotic cell death due to abrogation of MYC expression. Cancer Discovery 6 430-445. (https://doi. org/10.1158/2159-8290.CD-15-0754)

Patterson SG, Wei S, Chen X, Sallman DA, Gilvary DL, Zhong B, PowSang J, Yeatman T \& Djeu JY 2006 Novel role of Stat1 in the development of docetaxel resistance in prostate tumor cells. Oncogene 25 6113-6122. (https://doi.org/10.1038/sj.onc.1209632)

Puhr M, Hoefer J, Schafer G, Erb HH, Oh SJ, Klocker H, Heidegger I, Neuwirt H \& Culig Z 2012 Epithelial-to-mesenchymal transition leads to docetaxel resistance in prostate cancer and is mediated by reduced expression of miR-200c and miR-205. American Journal of
Pathology 181 2188-2201. (https://doi.org/10.1016/j. ajpath.2012.08.011)

Qin J, Lee HJ, Wu SP, Lin SC, Lanz RB, Creighton CJ, Demayo FJ, Tsai SY \& Tsai MJ 2014 Androgen deprivation-induced NCoA2 promotes metastatic and castration-resistant prostate cancer. Journal of Clinical Investigation 124 5013-5026. (https://doi.org/10.1172/JCI76412)

Ramos YF, Hestand MS, Verlaan M, Krabbendam E, Ariyurek Y, Van Galen M, Van Dam H, Van Ommen GJ, Den Dunnen JT, Zantema A, et al. 2010 Genome-wide assessment of differential roles for p300 and CBP in transcription regulation. Nucleic Acids Research $\mathbf{3 8}$ 5396-5408. (https://doi.org/10.1093/nar/gkq184)

Santer FR, Hoschele PP, Oh SJ, Erb HH, Bouchal J, Cavarretta IT, Parson W, Meyers DJ, Cole PA \& Culig Z 2011 Inhibition of the acetyltransferases p300 and CBP reveals a targetable function for p300 in the survival and invasion pathways of prostate cancer cell lines. Molecular Cancer Therapeutics 10 1644-1655. (https://doi. org/10.1158/1535-7163.MCT-11-0182)

Tang W, Zhou W, Xiang L, Wu X, Zhang P, Wang J, Liu G, Zhang W, Peng Y, Huang X, et al. 2019 The p300/YY1/miR-500a-5p/HDAC2 signalling axis regulates cell proliferation in human colorectal cancer. Nature Communications 10 663. (https://doi.org/10.1038/ s41467-018-08225-3)

Tannock IF, De Wit R, Berry WR, Horti J, Pluzanska A, Chi KN, Oudard S, Theodore C, James ND, Turesson I, et al. 2004 Docetaxel plus prednisone or mitoxantrone plus prednisone for advanced prostate cancer. New England Journal of Medicine 351 1502-1512. (https://doi.org/10.1056/NEJMoa040720)

Thadani-Mulero M, Portella L, Sun S, Sung M, Matov A, Vessella RL, Corey E, Nanus DM, Plymate SR \& Giannakakou P 2014 Androgen receptor splice variants determine taxane sensitivity in prostate cancer. Cancer Research 74 2270-2282. (https://doi.org/10.1158/00085472.CAN-13-2876)

Vazquez R, Civenni G, Kokanovic A, Shinde D, Cantergiani J, Marchetti M, Zoppi G, Ruggeri B, Liu PCC, Carbone GM, et al. 2019 Efficacy of novel bromodomain and extraterminal inhibitors in combination with chemotherapy for castration-resistant prostate cancer. European Urology Oncology [epub]. (https://doi.org/10.1016/j.euo.2019.07.013)

Wang YC, Wu YS, Hung CY, Wang SA, Young MJ, Hsu TI \& Hung JJ 2018 USP24 induces IL-6 in tumor-associated microenvironment by stabilizing p300 and beta-TrCP and promotes cancer malignancy. Nature Communications 9 3996. (https://doi.org/10.1038/s41467-01806178-1)

Zhu ML, Horbinski CM, Garzotto M, Qian DZ, Beer TM \& Kyprianou N 2010 Tubulin-targeting chemotherapy impairs androgen receptor activity in prostate cancer. Cancer Research 70 7992-8002. (https:// doi.org/10.1158/0008-5472.CAN-10-0585)

Received in final form 10 January 2020

Accepted 17 January 2020

Accepted Manuscript published online 17 January 2020 (c) 2020 The authors Published by Bioscientifica Ltd. Printed in Great Britain

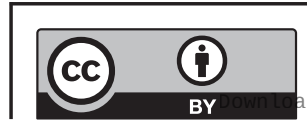

This work is licensed under a Creative Commons Attribution 4.0 International License. ded from Bioscientifica.com at 04/26/2023 09:57:30AM 